\title{
Caracterização estrutural em uma floresta de terra firme no estado do Amapá, Brasil
}

Anderson Pedro Bernardina Batista ${ }^{1 *}$, Wegliane Campelo da Silva Aparício ${ }^{2}$, Perseu da Silva Aparício ${ }^{2}$, Vanessa Silva dos Santos ${ }^{4}$, Robson Borges de Lima², José Marcio de Mello ${ }^{1}$

${ }^{1}$ Universidade Federal de Lavras, Departamento de Ciências Florestais, CEP 37200-000, Lavras, MG, Brasil

2Universidade do Estado do Amapá, Campus I, CEP 68900-000, Centro, Macapá, AP, Brasil

3Universidade Federal do Amapá, Campus Marco Zero, CEP 68903-419, Macapá, AP, Brasil

${ }^{4}$ Universidade Federal Rural de Pernambuco, Departamento de Ciência Florestal, CEP 52171-900, Dois Irmãos, Recife, PE, Brasil

"Autor correspondente:

anderson_pedro22@yahoo.com.br

Termos para indexação:

Fitossociologia

Florística

Distribuição espacial

Index terms:

Phytosociology

Floristics

Spatial distribution

Histórico do artigo:

Recebido em 13/04/2014

Aprovado em 19/03/2015

Publicado em 31/03/2015

doi: 10.4336/2015.pfb.35.81.689
Resumo - O objetivo deste trabalho foi caracterizar a composição florística, estrutura e distribuição espacial das espécies arbóreas em uma floresta de terra firme no Estado do Amapá. Foram coletados dados dendrométricos e florísticos em 200 unidades amostrais terciárias, sendo analisadas as árvores com diâmetro a 1,30 m do solo (DAP) $\geq 40$ $\mathrm{cm}$. Foram calculados parâmetros fitossociológicos, distribuição espacial e índice de diversidade de Shannon. No levantamento foram amostrados 4.030 indivíduos arbóreos, distribuídos em 37 famílias e 201 espécies, sendo Goupia glabra (cupiúba) a espécie de maior valor de importância (VI). Aárea florestal apresenta características de floresta preservada. A amostragem revelou que a vegetação arbórea ocorre com maior proporção de espécies com distribuição espacial aleatória, no entanto, as espécies como maior VI se distribuíram de forma agregada na área.

\section{Structural characterization in a upland forest in the state of Amapá, Brazil}

\begin{abstract}
The aim of this study was to characterize the floristic composition, structure and spatial distribution of tree species in an upland forest in the state of Amapá, Brazil. Dendrometric and floristic data were collected at 200 tertiary sampling units. It were analyzed data from trees with diameter at $1.30 \mathrm{~m}$ above soil level $(\mathrm{DBH}) \geq 40 \mathrm{~cm}$. Phytosociology, spatial distribution and Shannon diversity index were calculated. It were surveyed 4,030 individual trees, distributed in 37 families and 201 species. Goupia glabra (cupiúba) was the species with highest importance value (IV). The forest area presented characteristics of preserved forest. Sampling revealed that tree species occur with a higher proportion of species with random spatial distribution, however, the species with higher VI had aggregate distribution in the area.
\end{abstract}




\section{Introdução}

O estado do Amapá possui uma área de 143,4 mil $\mathrm{km}^{2}$, sendo $71,86 \%$ compostos por floresta densa de terra firme, que é o tipo de vegetação mais representativo da região (Rabelo, 2008). Dentre as características dessa vegetação, destacam-se a alta diversidade por unidade de área e vegetação de alto porte. Em termos de proteção florestal, esse estado é privilegiado, sendo considerado o mais preservado do Brasil, com 19 unidades de conservação, sendo 12 federais, cinco estaduais e duas municipais, representando $62 \%$ do seu território. Dentre as florestas públicas do Estado, destaca-se a Floresta Estadual do Amapá (FLOTA/AP) que representa um instrumento eficaz de política pública para o desenvolvimento econômico e uso sustentável dos recursos florestais (Amapá, 2009, 2013).

Antes de qualquer plano de ação, é necessário conhecer a composição e a estrutura florestal, pois essa informação é indispensável para a elaboração de planos de manejo sustentável (Costa Junior et al., 2008). Neste contexto, a análise fitossociológica possibilita melhor conhecimento e entendimento das espécies que compõem a floresta, possibilitando um melhor planejamento para o estabelecimento de estratégias adequadas para a conservação e manejo. Além disso, práticas de exploração desordenada dos recursos naturais das florestas podem ocasionar a perda da biodiversidade, entre outros impactos negativos. Neste sentido, o objetivo geral deste trabalho foi analisar a composição florística, estrutura e distribuição espacial das espécies arbóreas na floresta de terra firme no estado do Amapá, módulo pertencente a Floresta Estadual do Amapá (FLOTA/AP).

\section{Material e métodos}

A área de estudo compreende o módulo III da Floresta Estadual do Amapá, cuja extensão é de 742.960 ha. A área abrange os municípios Pedra Branca, Porto Grande, Ferreira Gomes, Tartarugalzinho, Pracuúba, Amapá e Calçoene, em proporções diferentes, conforme o Plano Anual de Outorga Florestal (PAOF) (Amapá, 2009, 2013). Segundo a classificação do IBGE (2012) a vegetação predominante nesta área é constituída pelas tipologias: Floresta de Terra Firme Densa de Baixo Platô (FTFDBP), Floresta de Terra Firme Densa Submontana (FTFDSm) e Transição Cerrado Floresta (TCF). O clima da região é do tipo Af, quente-úmido, com chuvas em todas as estações do ano, segundo a classificação de Köppen, conforme (Kottek et al., 2006). A temperatura média para todo o estado é em torno de $25^{\circ} \mathrm{C}$, em que as médias máximas e mínimas atingem, respectivamente, $32{ }^{\circ} \mathrm{C}$ e $22{ }^{\circ} \mathrm{C}$, e a precipitação média anual na região é de $2.284 \mathrm{~mm}$ (Drummond et al., 2008).

Foram utilizados dados dendrométricos e florísticos do inventário realizado na Floresta Estadual do Amapá, obtido no Instituto Estadual de Florestas do Amapá (IEF/ AP). Foram implantadas 10 unidades primárias (UP) de 8 ha cada, totalizando um universo amostral de 80 ha. Essas estão divididas em cinco unidades secundárias (US) que são subdivididas em quatro unidades terciárias (UT) de 20 × $200 \mathrm{~m}$, que foram sorteadas e distribuídas de forma aleatória na floresta, compreendendo as tipologias florestais: Floresta de Terra Firme Densa de Baixo Platô (FTFDBP) com oito UP; Floresta de Terra Firme Densa Submontana (FTFDSm) com uma UP; e Transição Cerrado Floresta (TCF) também uma UP.

Dentro de cada UT foram mensurados todos os indivíduos arbóreos com diâmetro a $1,30 \mathrm{~m}$ do solo (DAP) $\geq 40 \mathrm{~cm}$. Foram coletados materiais botânicos para a identificação, as exsicatas foram incorporadas ao Herbário da Universidade Federal Rural da Amazônia e foram classificadas segundo o sistema APG II (Angiosperm Phylogeny Group, 2003). A nomenclatura científica foi atualizada conforme Missouri Botanical Garden (Trópicos, 2014).

Foram calculados para todas as espécies arbóreas adultas os seguintes parâmetros fitossociológicos: densidade absoluta e relativa (DA e DR); frequência absoluta e relativa (FA e FR); dominância absoluta e relativa (DoA e DoR); valor de importância (VI); e valor de cobertura (VC), conforme Felfili \& Resende (2003). Os cálculos foram realizados por meio do software Mata Nativa 2 (Cientec, 2006) e pacote vegan (Oksanen et al., 2013) na plataforma R (R Core Team, 2013). É importante destacar que para os cálculos foram consideradas as UT que totalizaram $n=200$.

A diversidade de espécies foi analisada empregandose o índice de diversidade de Shannon (H'), considerando maior a diversidade florística, quanto maior o valor de H'. O Índice de Pielou (J') foi empregado para medir a proporção da diversidade observada em relação à máxima diversidade esperada. Os valores podem variar de 0 a 1 , sendo que, quanto mais próximo de 1 , maior a uniformidade do local (Felfili \& Resende, 2003). Estes índices foram calculados com auxílio do software Mata 
Nativa 2 (Cientec, 2006) e pacote vegan (Oksanen et al., 2013) na plataforma R (R Core Team, 2013).

A similaridade florística entre as tipologias foi avaliada por meio da técnica de agrupamento (cluster). Para análise de agrupamento foi aplicada a distância euclidiana como medida de dissimilaridade e o algoritmo de Ward para delimitação dos grupos, conforme Souza et al. (1997) e Batista et al. (2013). O processamento foi realizado com auxílio do pacote vegan (Oksanen et al., 2013) na plataforma R (R Core Team, 2013) e software Statistica 8 (Statsoft, 2012).

Para avaliar a distribuição espacial das espécies foi utilizado o índice de Payandeh (Pi), dado pela razão da variância do número de indivíduos, por unidade amostral, e a média do número de indivíduos. Para o cálculo utilizou-se o software Mata Nativa 2 (Cientec, 2006).

\section{Resultados e discussão}

No levantamento foram amostrados 4.030 indivíduos arbóreos, pertencentes a 37 famílias, 118 gêneros e 201 espécies. Dessas, 44 foram identificadas apenas em nível de gênero e 2 não foram identificadas (Tabela 1).

As famílias com maior número de espécies foram: Fabaceae (46), seguida por Sapotaceae (19), Lecythidaceae (12), Lauraceae (9), Meliaceae (8), Vochysiaceae (8), Apocynaceae (7), Chrysobalanaceae (7), Moraceae (7), Burseraceae (6), Malvaceae (6), Myristicaceae (6) e Urticaceae (6), correspondendo a $73,13 \%$ da riqueza total. Resultados semelhantes foram encontrados por Pereira et al. (2011) em 1,1 ha de terra firme na Reserva de Desenvolvimento Sustentável Rio Iratapuru no Amapá, onde encontraram 623 indivíduos distribuídos em 101 espécies, agrupadas em 32 famílias botânicas, sendo Fabaceae, Lauraceae, Sapotaceae e Lecythidaceae as que apresentaram maior riqueza, responsáveis por $61,6 \%$ do total das espécies amostradas. Pereira et al. (2007) em seu estudo na FLONA do Amapá, também encontraram a família Fabaceae com maior percentual de espécies.

Das 37 famílias encontradas neste estudo, 11 (29,72\%) apresentaram apenas uma espécie, foram elas: Araliaceae, Bixaceae, Boraginaceae, Calophyllaceae, Dichapelaceae, Goupiaceae, Lacistemataceae, Rubiaceae, Salicaceae, Simaroubacea e Siparunaceae. Possivelmente, essas famílias são constituídas de poucas espécies na área ou o nível de inclusão de DAP $\geq 40 \mathrm{~cm}$ adotado neste trabalho restringiu a inclusão de outros representantes dessas famílias. Outra possível explicação seria a substituição de espécies dessas famílias no processo sucessional natural em áreas de florestas maduras.

Foi observado que das 201 espécies amostradas na área de estudo, $58(28,85 \%)$ estiveram presentes nas três tipologias florestais estudadas (TCF, FTFDSm e FTFDBP). Na tipologia TCF foram encontradas 104 espécies, sendo que cinco foram exclusivas desta tipologia. Das 128 espécies amostradas na FTFDSm, quatro ocorreram exclusivamente nesta tipologia e, das 188 espécies encontradas na FTFDBP, 57 foram encontradas apenas nessa tipologia.

Considerando a diversidade florística, o índice de Shannon (H') calculado foi de 4,61 nats ind. ${ }^{-1}$. Esse valor foi superior ao encontrado por Pereira et al. (2007) na Floresta Nacional do Amapá $\left(4,07\right.$ nats ind. $\left.{ }^{-1}\right)$, e por Francez et al. (2007) que estudou mudanças ocorridas na composição florística em decorrência da exploração florestal, no Pará, antes da exploração (4,29 nats ind. $\left.{ }^{-1}\right)$ e após $\left(4,27\right.$ nats ind..$\left.^{-1}\right)$. Portanto, conforme o índice de Shannon encontrado, a área estudada apresenta uma relevante diversidade, podendo-se inferir que a mesma sofreu pouca perturbação. O índice de Pielou (J') encontrado para a área de estudo foi de 0,88 demonstrando uma boa uniformidade na distribuição do número de indivíduos pelo número de espécies.

Os valores de diversidade (H') e equabilidade (J') exibiram proximidade para as diferentes tipologias florestais estudadas: Floresta de Terra Firme Densa de Baixo Platô (FTFDBP) $\left(\mathrm{H}^{\prime}=4,57\right.$ nats ind. $\left.{ }^{-1}, \mathrm{~J}^{\prime}=0,87\right)$, Floresta de Terra Firme Densa Submontana (FTFDSm) $\left(\mathrm{H}^{\prime}=4,10\right.$ nats ind. $\left.{ }^{-1}, \mathrm{~J}^{\prime}=0,90\right)$ e transição Cerrado Floresta (TCF) $\left(\mathrm{H}^{\prime}=4,18\right.$ nats ind. $\left..^{-1}, \mathrm{~J}^{\prime}=0,90\right)$.

Na Figura 1 é possível verificar a formação de dois grupos, com maior similaridade florística entre FTFDSm e TCF, formando de forma explicita um grupo, enquanto que a FTFDBP demonstrou menor semelhança das demais, ficando isolada em outro grupo. Esse fato pode ser explicado pela maior riqueza e diversidade encontrada para a FTFDBP, visto que nessa tipologia foram registradas 188 espécies, sendo 57 exclusivas, contribuindo com maior riqueza de espécies para a área florestal. 


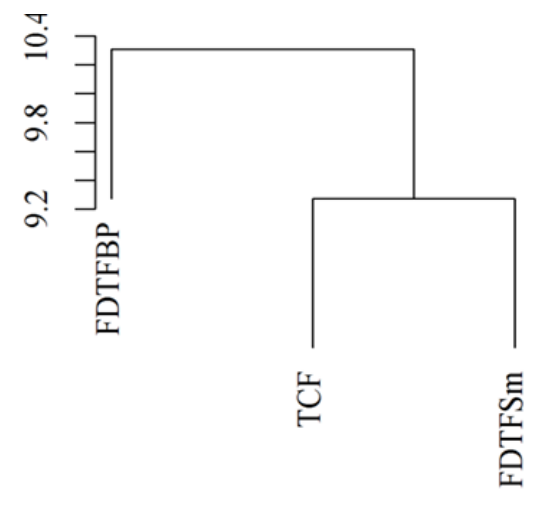

Figura 1. Dendrograma gerado a partir da análise agrupamento, por meio do método de Ward e distância euclidiana, para as tipologias: $\mathrm{TCF}=$ Transição cerrado/ floresta, FTFDSm=Floresta de terra firme densa submontana e FTFDBP=Floresta de terra firme densa de baixo platô.

Em termos de abundância, as espécies que se destacaram foram: Goupia glabra (cupiúba) com 237 indivíduos em toda amostragem, seguida de Eschweilera sp. (matamatá vermelho), com 160 indivíduos, Carapa guianensis (andiroba) com 152 indivíduos, Eschweilera coriacea (matamatá branco) com 134 indivíduos e Vochysia sp.1 (quaruba), com 126 indivíduos. Essas espécies foram amostradas nas três tipologias estudadas. Alves \& Miranda (2008), analisando a estrutura de uma floresta de terra firme no Pará, também encontraram Goupia glabra como a mais abundante, com 4.305 indivíduos. Vale salientar que 25 espécies $(12,43 \%)$ apresentaram apenas um indivíduo $(\mathrm{DA}=0,01)$, dentre elas: Zygia ramiflora, Pouteria sp.3, Eriotheca longipedicellata, Eperua sp., Oxandra acuminata, Protium pilosum, entre outras (Tabela 1). Essas espécies são consideradas por Oliveira et al. (2008) como "localmente raras" e merecem atenção do ponto de vista conservacionista. Espécies com baixa abundância local apresentam maior risco de extinção por estocasticidade demográfica, ambiental ou genética (Alves \& Miranda, 2008).

As espécies que apresentaram maiores valores para densidade relativa, em ordem decrescente, foram: Goupia glabra, Eschweilera sp., Carapa guianensis, Eschweilera coriacea e Vochysia sp.1, representando $14,51 \%$ do total (Tabela 1).

Tabela 1. Florísitca e parâmetros fitossociológicos dos indivíduos arbóreos amostrados na floresta de terra firme no Amapá.

\begin{tabular}{|c|c|c|c|c|c|c|c|c|c|c|c|c|}
\hline Espécie & DA & DR & FA & FR & DoA & DoR & $\mathbf{V C}$ & VI & $\mathbf{P i}$ & TCF & FTFDSm & FTFDBP \\
\hline Goupia glabra & 2,16 & 4,25 & 45,5 & 3,06 & 1,09 & 6,41 & 10,7 & 13,7 & A & $\mathrm{X}$ & $\mathrm{X}$ & $\mathrm{X}$ \\
\hline Vochysia sp.1 & 1,15 & 2,26 & 29,8 & 2,01 & 0,55 & 3,22 & 5,48 & 7,49 & A & $\mathrm{X}$ & $\mathrm{X}$ & $\mathrm{X}$ \\
\hline Carapa guianensis & 1,38 & 2,73 & 37,8 & 2,55 & 0,37 & 2,17 & 4,89 & 7,44 & $\mathrm{TA}$ & $\mathrm{X}$ & $\mathrm{X}$ & $X$ \\
\hline Dinizia excelsa & 0,57 & 1,13 & 16,7 & 1,13 & 0,83 & 4,88 & 6,01 & 7,14 & A & $\mathrm{X}$ & $\mathrm{X}$ & $\mathrm{X}$ \\
\hline Eschweilera sp. & 1,46 & 2,87 & 31,6 & 2,13 & 0,36 & 2,13 & 5 & 7,13 & A & $\mathrm{X}$ & $\mathrm{X}$ & $\mathrm{X}$ \\
\hline Eschweilera coriacea & 1,22 & 2,4 & 30,6 & 2,06 & 0,29 & 1,7 & 4,1 & 6,15 & A & $\mathrm{X}$ & $\mathrm{X}$ & $\mathrm{X}$ \\
\hline Minquartia guianensis & 1,05 & 2,06 & 28,7 & 1,93 & 0,34 & 1,97 & 4,04 & 5,97 & A & $\mathrm{X}$ & $\mathrm{X}$ & $\mathrm{X}$ \\
\hline Aspidosperma excelsum & 0,85 & 1,67 & 24,7 & 1,66 & 0,43 & 2,55 & 4,21 & 5,88 & $\mathrm{TA}$ & $X$ & $X$ & $X$ \\
\hline Conceveiba guianensis & 0,88 & 1,74 & 28,7 & 1,93 & 0,37 & 2,17 & 3,91 & 5,84 & $\mathrm{TA}$ & $\mathrm{X}$ & $\mathrm{X}$ & $\mathrm{X}$ \\
\hline Micropholis acutangula & 1,12 & 2,21 & 24 & 1,62 & 0,33 & 1,95 & 4,16 & 5,77 & A & $\mathrm{X}$ & $\mathrm{X}$ & $\mathrm{X}$ \\
\hline Nectandra rubra & 0,86 & 1,69 & 23,6 & 1,59 & 0,4 & 2,37 & 4,06 & 5,65 & A & $\mathrm{X}$ & $\mathrm{X}$ & $\mathrm{X}$ \\
\hline Protium decandrum & 1,04 & 2,04 & 28,4 & 1,91 & 0,29 & 1,7 & 3,74 & 5,65 & A & $\mathrm{X}$ & $\mathrm{X}$ & $\mathrm{X}$ \\
\hline Licania canescens & 1,04 & 2,04 & 29,8 & 2,01 & 0,24 & 1,44 & 3,48 & 5,49 & $\mathrm{TA}$ & $\mathrm{X}$ & $\mathrm{X}$ & $\mathrm{X}$ \\
\hline Eschweilera pedicellata & 1,06 & 2,1 & 21,1 & 1,42 & 0,31 & 1,83 & 3,93 & 5,35 & A & & $\mathrm{X}$ & $\mathrm{X}$ \\
\hline Protium tenuifolium & 1 & 1,97 & 25,1 & 1,69 & 0,27 & 1,6 & 3,58 & 5,27 & A & $\mathrm{X}$ & $\mathrm{X}$ & $\mathrm{X}$ \\
\hline Manilkara huberi & 0,81 & 1,6 & 24,7 & 1,66 & 0,34 & 1,97 & 3,57 & 5,24 & $\mathrm{~T} A$ & $\mathrm{X}$ & $X$ & $X$ \\
\hline
\end{tabular}

Valores apresentados em ordem decrescente de valor de importância (VI). DA = densidade absoluta (ind. ha $\left.{ }^{-1}\right)$; DR $=$ densidade relativa (\%); FA= frequência absoluta; $\mathrm{FR}=$ frequência relativa; $\mathrm{DoA}=$ dominância absoluta $\left(\mathrm{m}^{2} \mathrm{ha}^{-1}\right) ; \mathrm{DoR}=$ dominância relativa; $\mathrm{VC}=$ valor de cobertura; $\mathrm{VI}=$ valor de importância e $\mathrm{Pi}$ = índice de Payandeh ( $\mathrm{A}=$ agrupamento; $\mathrm{T} \mathrm{A}=$ tendência agrupamento; $\mathrm{N} \mathrm{A}=$ não agrupada $), \mathrm{NI}=$ não identificada. TCF $=$ transição Cerrado Floresta, FTFDSm = Floresta de Terra Firme Densa Submontana e FTFDBP = Floresta de Terra Firme Densa de Baixo Platô. 
Tabela 1. Continuação.

\begin{tabular}{|c|c|c|c|c|c|c|c|c|c|c|c|c|}
\hline Espécie & DA & DR & FA & FR & DoA & DoR & $\mathrm{VC}$ & VI & $\mathbf{P i}$ & TCF & FTFDSm & FTFDBP \\
\hline Tachigali mymercophila & 0,76 & 1,49 & 25,1 & 1,69 & 0,28 & 1,67 & 3,16 & 4,85 & $\mathrm{TA}$ & $\mathrm{X}$ & $\mathrm{X}$ & $\mathrm{X}$ \\
\hline Tabebuia serratifolia & 0,81 & 1,6 & 24 & 1,62 & 0,27 & 1,57 & 3,16 & 4,78 & $\mathrm{~T} A$ & $\mathrm{X}$ & $\mathrm{X}$ & $\mathrm{X}$ \\
\hline Vouacapoua americana & 0,96 & 1,88 & 20 & 1,35 & 0,24 & 1,41 & 3,29 & 4,64 & A & & $\mathrm{X}$ & $\mathrm{X}$ \\
\hline Couratari guianensis & 0,68 & 1,35 & 21,5 & 1,44 & 0,31 & 1,82 & 3,16 & 4,6 & $\mathrm{~T} A$ & $\mathrm{X}$ & $\mathrm{X}$ & $\mathrm{X}$ \\
\hline Sloanea grandiflora & 0,79 & 1,56 & 26,2 & 1,76 & 0,22 & 1,27 & 2,83 & 4,6 & $\mathrm{~T} A$ & & $\mathrm{X}$ & $\mathrm{X}$ \\
\hline Dipteryx odorata & 0,65 & 1,27 & 22,2 & 1,49 & 0,28 & 1,62 & 2,9 & 4,39 & $\mathrm{~T} A$ & $\mathrm{X}$ & $\mathrm{X}$ & $\mathrm{X}$ \\
\hline Lecythis lurida & 0,8 & 1,58 & 22,9 & 1,54 & 0,21 & 1,24 & 2,82 & 4,37 & $\mathrm{~T} A$ & $\mathrm{X}$ & $\mathrm{X}$ & $\mathrm{X}$ \\
\hline Inga auristellae & 0,77 & 1,52 & 23,6 & 1,59 & 0,21 & 1,21 & 2,73 & 4,32 & $\mathrm{~T} A$ & $\mathrm{X}$ & $\mathrm{X}$ & $\mathrm{X}$ \\
\hline $\begin{array}{l}\text { Guarea pubescens } \\
\text { subsp. Pubiflora }\end{array}$ & 0,72 & 1,42 & 22,6 & 1,52 & 0,22 & 1,3 & 2,72 & 4,23 & $\mathrm{~T} A$ & $\mathrm{X}$ & $\mathrm{X}$ & $\mathrm{X}$ \\
\hline Alexa grandiflora & 0,67 & 1,33 & 21,5 & 1,44 & 0,23 & 1,33 & 2,66 & 4,1 & T A & $\mathrm{X}$ & $\mathrm{X}$ & $\mathrm{X}$ \\
\hline Pouteria caimito & 0,82 & 1,61 & 20,7 & 1,4 & 0,18 & 1,07 & 2,69 & 4,08 & $\mathrm{~A}$ & $\mathrm{X}$ & $\mathrm{X}$ & $\mathrm{X}$ \\
\hline Abarema jupunba & 0,69 & 1,36 & 21,5 & 1,44 & 0,21 & 1,25 & 2,61 & 4,06 & $\mathrm{~T} A$ & & $\mathrm{X}$ & $\mathrm{X}$ \\
\hline Qualea albiflora & 0,6 & 1,18 & 18,6 & 1,25 & 0,26 & 1,55 & 2,74 & 3,99 & $\mathrm{~T} A$ & $\mathrm{X}$ & & $\mathrm{X}$ \\
\hline Bowdichia nitida & 0,65 & 1,27 & 20 & 1,35 & 0,18 & 1,04 & 2,32 & 3,66 & $\mathrm{~T}$ A & $X$ & $X$ & $\mathrm{X}$ \\
\hline $\begin{array}{l}\text { Pseudopiptadenia } \\
\text { psylostachya }\end{array}$ & 0,56 & 1,11 & 18,9 & 1,27 & 0,22 & 1,27 & 2,38 & 3,65 & T A & & $\mathrm{X}$ & $\mathrm{X}$ \\
\hline Protium altsonii & 0,66 & 1,31 & 16,7 & 1,13 & 0,18 & 1,04 & 2,34 & 3,47 & A & $X$ & $X$ & $X$ \\
\hline Eperua rubiginosa & 0,68 & 1,35 & 10,9 & 0,73 & 0,22 & 1,29 & 2,64 & 3,37 & A & $\mathrm{X}$ & $\mathrm{X}$ & $\mathrm{X}$ \\
\hline Micropholis venulosa & 0,56 & 1,09 & 15,6 & 1,05 & 0,2 & 1,16 & 2,26 & 3,31 & A & $\mathrm{X}$ & $\mathrm{X}$ & $\mathrm{X}$ \\
\hline Neoxythece elegans & 0,62 & 1,22 & 17,1 & 1,15 & 0,14 & 0,83 & 2,05 & 3,2 & $\mathrm{~A}$ & $\mathrm{X}$ & $\mathrm{X}$ & $\mathrm{X}$ \\
\hline $\begin{array}{l}\text { Osteophloeum } \\
\text { platyspermum }\end{array}$ & 0,56 & 1,09 & 15,3 & 1,03 & 0,17 & 1 & 2,1 & 3,13 & A & $\mathrm{X}$ & $\mathrm{X}$ & $\mathrm{X}$ \\
\hline Hymenaea courbaril & 0,46 & 0,9 & 13,5 & 0,91 & 0,16 & 0,95 & 1,85 & 2,75 & A & $\mathrm{X}$ & $\mathrm{X}$ & $\mathrm{X}$ \\
\hline Symphonia globulifera & 0,46 & 0,91 & 15,3 & 1,03 & 0,12 & 0,7 & 1,62 & 2,65 & $\mathrm{~T} A$ & $\mathrm{X}$ & $\mathrm{X}$ & $\mathrm{X}$ \\
\hline Pterocarpus amazonicus & 0,49 & 0,97 & 12,4 & 0,83 & 0,14 & 0,84 & 1,81 & 2,65 & A & & $X$ & $\mathrm{X}$ \\
\hline Iryanthera paraenses & 0,46 & 0,91 & 13,5 & 0,91 & 0,13 & 0,77 & 1,69 & 2,6 & A & & $X$ & $\mathrm{X}$ \\
\hline $\begin{array}{l}\text { Micropholis } \\
\text { longipedicellata }\end{array}$ & 0,49 & 0,97 & 11,6 & 0,78 & 0,13 & 0,77 & 1,73 & 2,52 & $\mathrm{~A}$ & $\mathrm{X}$ & $\mathrm{X}$ & $\mathrm{X}$ \\
\hline Ocotea sp. 2 & 0,4 & 0,79 & 14,6 & 0,98 & 0,12 & 0,69 & 1,48 & 2,46 & $\mathrm{~T}$ A & & & $\mathrm{X}$ \\
\hline Mouriri apiranga & 0,42 & 0,82 & 11,3 & 0,76 & 0,11 & 0,65 & 1,47 & 2,23 & A & $\mathrm{X}$ & $\mathrm{X}$ & $\mathrm{X}$ \\
\hline Caryocar villosum & 0,26 & 0,5 & 10,2 & 0,69 & 0,17 & 1,03 & 1,53 & 2,21 & N A & $\mathrm{X}$ & $\mathrm{X}$ & $\mathrm{X}$ \\
\hline Virola michelii & 0,38 & 0,75 & 12 & 0,81 & 0,11 & 0,65 & 1,4 & 2,21 & $\mathrm{~T} A$ & & $\mathrm{X}$ & $\mathrm{X}$ \\
\hline Ecclinusa lanceolata & 0,43 & 0,84 & 12 & 0,81 & 0,1 & 0,56 & 1,4 & 2,21 & A & $\mathrm{X}$ & $\mathrm{X}$ & $\mathrm{X}$ \\
\hline Couma guianensis & 0,43 & 0,84 & 8,73 & 0,59 & 0,09 & 0,55 & 1,4 & 1,98 & A & $\mathrm{X}$ & & $\mathrm{X}$ \\
\hline Brosimum parinarioides & 0,3 & 0,59 & 10,6 & 0,71 & 0,11 & 0,63 & 1,22 & 1,93 & $\mathrm{~T}$ A & $\mathrm{X}$ & & $\mathrm{X}$ \\
\hline Manilkara sp. & 0,33 & 0,65 & 11,3 & 0,76 & 0,09 & 0,52 & 1,17 & 1,92 & $\mathrm{~T} A$ & $\mathrm{X}$ & $\mathrm{X}$ & $\mathrm{X}$ \\
\hline Iryanthera grandis & 0,34 & 0,66 & 11,3 & 0,76 & 0,08 & 0,48 & 1,15 & 1,91 & $\mathrm{~T} A$ & $\mathrm{X}$ & $\mathrm{X}$ & $\mathrm{X}$ \\
\hline
\end{tabular}

Valores apresentados em ordem decrescente de valor de importância (VI). DA = densidade absoluta (ind. ha $\left.{ }^{-1}\right)$; DR = densidade relativa (\%); FA= frequência absoluta; $\mathrm{FR}=$ frequência relativa; $\mathrm{DoA}=$ dominância absoluta $\left(\mathrm{m}^{2} \mathrm{ha}^{-1}\right) ; \mathrm{DoR}=$ dominância relativa; $\mathrm{VC}=$ valor de cobertura; $\mathrm{VI}=\mathrm{valor}$ de importância e $\mathrm{Pi}$ = índice de Payandeh $(\mathrm{A}=$ agrupamento; $\mathrm{T} \mathrm{A}=$ tendência agrupamento; $\mathrm{N} \mathrm{A}=$ não agrupada $), \mathrm{NI}=$ não identificada. $\mathrm{TCF}=$ transição Cerrado Floresta, FTFDSm = Floresta de Terra Firme Densa Submontana e FTFDBP = Floresta de Terra Firme Densa de Baixo Platô. 
Tabela 1. Continuação.

\begin{tabular}{|c|c|c|c|c|c|c|c|c|c|c|c|c|}
\hline Espécie & DA & DR & FA & FR & DoA & DoR & VC & VI & $\mathbf{P i}$ & TCF & FTFDSm & FTFDBP \\
\hline Licania octandra & 0,33 & 0,65 & 10,2 & 0,69 & 0,09 & 0,55 & 1,2 & 1,88 & $\mathrm{~T}$ A & $X$ & & $\mathrm{X}$ \\
\hline Hymenolobium petraeum & 0,22 & 0,43 & 7,64 & 0,51 & 0,16 & 0,92 & 1,35 & 1,87 & T A & $\mathrm{X}$ & $\mathrm{X}$ & $\mathrm{X}$ \\
\hline Caryocar glabrum & 0,22 & 0,43 & 8,36 & 0,56 & 0,14 & 0,83 & 1,26 & 1,82 & N A & $X$ & $X$ & $X$ \\
\hline Parinarium sp. & 0,3 & 0,59 & 10,2 & 0,69 & 0,09 & 0,53 & 1,12 & 1,81 & $\mathrm{TA}$ & & $\mathrm{X}$ & $\mathrm{X}$ \\
\hline Bixa arborea & 0,3 & 0,59 & 9,45 & 0,64 & 0,1 & 0,56 & 1,15 & 1,79 & $\mathrm{~T} A$ & & $\mathrm{X}$ & $\mathrm{X}$ \\
\hline Sloanea garckeana & 0,32 & 0,63 & 10,6 & 0,71 & 0,07 & 0,39 & 1,02 & 1,73 & T A & $\mathrm{X}$ & $\mathrm{X}$ & $\mathrm{X}$ \\
\hline Tachigali paniculata & 0,26 & 0,5 & 9,45 & 0,64 & 0,08 & 0,49 & 0,99 & 1,63 & $\mathrm{~T} \mathrm{~A}$ & & $\mathrm{X}$ & $\mathrm{X}$ \\
\hline Pachira sp. & 0,26 & 0,5 & 9,82 & 0,66 & 0,07 & 0,42 & 0,92 & 1,58 & NA & $\mathrm{X}$ & $\mathrm{X}$ & $\mathrm{X}$ \\
\hline Erisma uncinatum & 0,2 & 0,39 & 8 & 0,54 & 0,11 & 0,62 & 1,02 & 1,56 & NA & $\mathrm{X}$ & $\mathrm{X}$ & $\mathrm{X}$ \\
\hline Vantanea parviflora & 0,37 & 0,74 & 3,64 & 0,24 & 0,09 & 0,54 & 1,28 & 1,52 & A & & & $\mathrm{X}$ \\
\hline Simarouba amara & 0,26 & 0,5 & 8,73 & 0,59 & 0,06 & 0,36 & 0,86 & 1,45 & $\mathrm{~T}$ A & $\mathrm{X}$ & $X$ & $X$ \\
\hline Hymenaea parvifolia & 0,26 & 0,5 & 6,91 & 0,47 & 0,07 & 0,43 & 0,94 & 1,4 & A & & $\mathrm{X}$ & $\mathrm{X}$ \\
\hline Taralea oppositifolia & 0,25 & 0,48 & 8 & 0,54 & 0,06 & 0,35 & 0,84 & 1,37 & $\mathrm{~T} A$ & $\mathrm{X}$ & $\mathrm{X}$ & $\mathrm{X}$ \\
\hline $\begin{array}{l}\text { Geissospermum } \\
\text { sericeum }\end{array}$ & 0,24 & 0,47 & 8 & 0,54 & 0,06 & 0,36 & 0,82 & 1,36 & $\mathrm{~T}$ A & & & $\mathrm{X}$ \\
\hline Virola sp. & 0,23 & 0,45 & 8 & 0,54 & 0,06 & 0,37 & 0,82 & 1,36 & $\mathrm{~T} A$ & $\mathrm{X}$ & $\mathrm{X}$ & $\mathrm{X}$ \\
\hline Inga sp.1 & 0,25 & 0,48 & 8,36 & 0,56 & 0,05 & 0,29 & 0,78 & 1,34 & $\mathrm{~T}$ A & $\mathrm{X}$ & $\mathrm{X}$ & $\mathrm{X}$ \\
\hline Pouteria sp.4 & 0,24 & 0,47 & 8 & 0,54 & 0,05 & 0,3 & 0,76 & 1,3 & $\mathrm{~T} A$ & $\mathrm{X}$ & & $\mathrm{X}$ \\
\hline Cedrela odorata & 0,12 & 0,23 & 4,36 & 0,29 & 0,12 & 0,71 & 0,95 & 1,24 & $\mathrm{~T} A$ & & & $\mathrm{X}$ \\
\hline Hirtella sp. & 0,24 & 0,47 & 5,09 & 0,34 & 0,07 & 0,43 & 0,89 & 1,23 & A & $\mathrm{X}$ & $\mathrm{X}$ & $\mathrm{X}$ \\
\hline Couepia excelsa & 0,2 & 0,39 & 8 & 0,54 & 0,05 & 0,3 & 0,69 & 1,23 & NA & & & $\mathrm{X}$ \\
\hline Lecythis pisonis & 0,18 & 0,36 & 6,91 & 0,47 & 0,07 & 0,4 & 0,76 & 1,22 & $\mathrm{TA}$ & $\mathrm{X}$ & & $\mathrm{X}$ \\
\hline Dialium guianense & 0,2 & 0,39 & 7,64 & 0,51 & 0,05 & 0,31 & 0,71 & 1,22 & $\mathrm{~T}$ A & & $X$ & $X$ \\
\hline Peltogyne paniculata & 0,19 & 0,38 & 6,91 & 0,47 & 0,06 & 0,34 & 0,72 & 1,19 & $\mathrm{~T} A$ & $\mathrm{X}$ & $\mathrm{X}$ & $\mathrm{X}$ \\
\hline $\begin{array}{l}\text { Stryphnodendron } \\
\text { pulcherrimum }\end{array}$ & 0,19 & 0,38 & 7,64 & 0,51 & 0,05 & 0,29 & 0,67 & 1,19 & NA & $\mathrm{X}$ & $\mathrm{X}$ & $\mathrm{X}$ \\
\hline Guatteria sp. & 0,19 & 0,38 & 7,64 & 0,51 & 0,04 & 0,24 & 0,62 & 1,13 & NA & & $\mathrm{X}$ & $\mathrm{X}$ \\
\hline Apeiba tibourbou & 0,17 & 0,34 & 6,91 & 0,47 & 0,05 & 0,3 & 0,64 & 1,11 & NA & $\mathrm{X}$ & $\mathrm{X}$ & $\mathrm{X}$ \\
\hline Pseudolmedia sp. & 0,17 & 0,34 & 6,91 & 0,47 & 0,05 & 0,27 & 0,61 & 1,08 & NA & & $\mathrm{X}$ & $X$ \\
\hline Parkia multijuga & 0,16 & 0,32 & 5,09 & 0,34 & 0,07 & 0,41 & 0,73 & 1,07 & $\mathrm{~T}$ A & $\mathrm{X}$ & $\mathrm{X}$ & $\mathrm{X}$ \\
\hline Pourouma heterophylla & 0,18 & 0,36 & 6,55 & 0,44 & 0,03 & 0,18 & 0,54 & 0,98 & $\mathrm{~T} A$ & & & $\mathrm{X}$ \\
\hline Tachigali sp. & 0,16 & 0,3 & 5,82 & 0,39 & 0,05 & 0,27 & 0,57 & 0,96 & $\mathrm{~T}$ A & & $X$ & $X$ \\
\hline Terminalia amazonia & 0,16 & 0,32 & 5,45 & 0,37 & 0,05 & 0,27 & 0,59 & 0,96 & $\mathrm{~T} A$ & $\mathrm{X}$ & & $\mathrm{X}$ \\
\hline Lecythis sp. & 0,15 & 0,29 & 5,45 & 0,37 & 0,05 & 0,27 & 0,55 & 0,92 & $\mathrm{~T}$ A & & & $\mathrm{X}$ \\
\hline Pentaclethra macroloba & 0,19 & 0,38 & 4 & 0,27 & 0,04 & 0,24 & 0,62 & 0,89 & A & $\mathrm{X}$ & & $\mathrm{X}$ \\
\hline Inga velutina & 0,17 & 0,34 & 5,09 & 0,34 & 0,03 & 0,2 & 0,54 & 0,88 & $\mathrm{~T}$ A & & $\mathrm{X}$ & $\mathrm{X}$ \\
\hline Anacardium giganteum & 0,12 & 0,23 & 4 & 0,27 & 0,06 & 0,38 & 0,61 & 0,88 & $\mathrm{~T}$ A & $X$ & $X$ & $X$ \\
\hline Terminalia sp. & 0,14 & 0,27 & 5,45 & 0,37 & 0,04 & 0,24 & 0,51 & 0,88 & NA & $\mathrm{X}$ & & $\mathrm{X}$ \\
\hline
\end{tabular}

Valores apresentados em ordem decrescente de valor de importância $(\mathrm{VI}) . \mathrm{DA}=$ densidade absoluta (ind. ha $\left.{ }^{-1}\right)$; $\mathrm{DR}=$ densidade relativa $(\%)$; $\mathrm{FA}=$ frequência absoluta; $\mathrm{FR}=$ frequência relativa; $\mathrm{DoA}=$ dominância absoluta $\left(\mathrm{m}^{2} \mathrm{ha}^{-1}\right) ; \mathrm{DoR}=$ dominância relativa; $\mathrm{VC}=$ valor de cobertura; $\mathrm{VI}=$ valor de importância e $\mathrm{Pi}$ = índice de Payandeh $(\mathrm{A}=$ agrupamento; $\mathrm{T} \mathrm{A}$ = tendência agrupamento; $\mathrm{N} \mathrm{A}=$ não agrupada), $\mathrm{NI}=$ não identificada. $\mathrm{TCF}=$ transição Cerrado Floresta, FTFDSm = Floresta de Terra Firme Densa Submontana e FTFDBP = Floresta de Terra Firme Densa de Baixo Platô. 
Tabela 1. Continuação.

\begin{tabular}{|c|c|c|c|c|c|c|c|c|c|c|c|c|}
\hline Espécie & DA & DR & FA & FR & DoA & DoR & VC & VI & $\mathbf{P i}$ & TCF & FTFDSm & FTFDBP \\
\hline Inga sp.2 & 0,16 & 0,32 & 5,45 & 0,37 & 0,03 & 0,18 & 0,5 & 0,87 & T A & & $\mathrm{X}$ & $\mathrm{X}$ \\
\hline Pourouma sp. & 0,15 & 0,29 & 5,82 & 0,39 & 0,03 & 0,17 & 0,46 & 0,85 & N A & & $\mathrm{X}$ & $\mathrm{X}$ \\
\hline Tabebuia sp. & 0,13 & 0,25 & 4,36 & 0,29 & 0,05 & 0,29 & 0,54 & 0,84 & $\mathrm{TA}$ & $\mathrm{X}$ & & $\mathrm{X}$ \\
\hline Qualea paraensis & 0,14 & 0,27 & 3,64 & 0,24 & 0,05 & 0,32 & 0,59 & 0,83 & $\mathrm{~A}$ & $\mathrm{X}$ & & $\mathrm{X}$ \\
\hline Vochysia maxima & 0,12 & 0,23 & 4,36 & 0,29 & 0,04 & 0,25 & 0,48 & 0,78 & $\mathrm{~T} A$ & $\mathrm{X}$ & $\mathrm{X}$ & $\mathrm{X}$ \\
\hline Ocotea sp.1 & 0,13 & 0,25 & 5,09 & 0,34 & 0,03 & 0,17 & 0,42 & 0,77 & $\mathrm{NA}$ & & & $\mathrm{X}$ \\
\hline Tabebuia impertiginosa & 0,11 & 0,22 & 4,36 & 0,29 & 0,04 & 0,25 & 0,47 & 0,76 & NA & $\mathrm{X}$ & $\mathrm{X}$ & $\mathrm{X}$ \\
\hline Brosimum sp. & 0,11 & 0,22 & 4,36 & 0,29 & 0,04 & 0,24 & 0,46 & 0,75 & NA & & $\mathrm{X}$ & $\mathrm{X}$ \\
\hline Jacaranda copaia & 0,13 & 0,25 & 4,73 & 0,32 & 0,03 & 0,17 & 0,42 & 0,74 & $\mathrm{~T} A$ & $\mathrm{X}$ & & $\mathrm{X}$ \\
\hline Aniba burchellii & 0,13 & 0,25 & 4,36 & 0,29 & 0,03 & 0,18 & 0,43 & 0,72 & $\mathrm{TA}$ & & $\mathrm{X}$ & $\mathrm{X}$ \\
\hline Vochysia guianensis & 0,1 & 0,2 & 3,64 & 0,24 & 0,04 & 0,26 & 0,46 & 0,7 & $\mathrm{TA}$ & & & $\mathrm{X}$ \\
\hline Bagassa guianensis & 0,1 & 0,2 & 4 & 0,27 & 0,04 & 0,22 & 0,42 & 0,69 & NA & & $\mathrm{X}$ & $\mathrm{X}$ \\
\hline Parkia pendula & 0,09 & 0,18 & 3,64 & 0,24 & 0,04 & 0,25 & 0,43 & 0,68 & $\mathrm{NA}$ & $\mathrm{X}$ & & $\mathrm{X}$ \\
\hline Naucleopsis sp. & 0,12 & 0,23 & 3,64 & 0,24 & 0,03 & 0,19 & 0,42 & 0,67 & $\mathrm{TA}$ & & & $\mathrm{X}$ \\
\hline Astronium lecontei & 0,1 & 0,2 & 3,64 & 0,24 & 0,04 & 0,22 & 0,42 & 0,66 & $\mathrm{~T} A$ & $\mathrm{X}$ & & $\mathrm{X}$ \\
\hline Parkia gigantocarpa & 0,1 & 0,2 & 4 & 0,27 & 0,03 & 0,19 & 0,39 & 0,66 & $\mathrm{NA}$ & & & $\mathrm{X}$ \\
\hline Eschweilera grandiflora & 0,12 & 0,23 & 4 & 0,27 & 0,03 & 0,16 & 0,39 & 0,66 & $\mathrm{~T} \mathrm{~A}$ & $\mathrm{X}$ & & $\mathrm{X}$ \\
\hline Pourouma ovata & 0,12 & 0,23 & 4,73 & 0,32 & 0,02 & 0,1 & 0,34 & 0,66 & NA & & $\mathrm{X}$ & $\mathrm{X}$ \\
\hline Zygia racemosa & 0,11 & 0,22 & 4,36 & 0,29 & 0,02 & 0,14 & 0,35 & 0,65 & NA & & $\mathrm{X}$ & $\mathrm{X}$ \\
\hline Cariniana sp. & 0,1 & 0,2 & 3,27 & 0,22 & 0,04 & 0,2 & 0,4 & 0,62 & A & $\mathrm{X}$ & & $\mathrm{X}$ \\
\hline Micropholis melinoniana & 0,11 & 0,22 & 3,27 & 0,22 & 0,03 & 0,19 & 0,4 & 0,62 & $\mathrm{TA}$ & $\mathrm{X}$ & & $\mathrm{X}$ \\
\hline Hevea brasiliensis & 0,1 & 0,2 & 3,27 & 0,22 & 0,03 & 0,2 & 0,4 & 0,62 & $\mathrm{~T}$ A & $\mathrm{X}$ & $\mathrm{X}$ & $\mathrm{X}$ \\
\hline Vochysia sp.2 & 0,08 & 0,16 & 2,55 & 0,17 & 0,04 & 0,21 & 0,37 & 0,54 & $\mathrm{TA}$ & & $X$ & $X$ \\
\hline Qualea retusa & 0,08 & 0,16 & 2,55 & 0,17 & 0,03 & 0,2 & 0,36 & 0,54 & $\mathrm{TA}$ & & & $\mathrm{X}$ \\
\hline Licaria cannella & 0,09 & 0,18 & 3,64 & 0,24 & 0,02 & 0,11 & 0,29 & 0,53 & N A & & $\mathrm{X}$ & $\mathrm{X}$ \\
\hline Diospyros sp.1 & 0,08 & 0,16 & 3,27 & 0,22 & 0,03 & 0,15 & 0,31 & 0,53 & N A & & $\mathrm{X}$ & $\mathrm{X}$ \\
\hline Poecilanthe effusa & 0,09 & 0,18 & 2,91 & 0,2 & 0,02 & 0,13 & 0,31 & 0,5 & $\mathrm{~T}$ A & $\mathrm{X}$ & & $\mathrm{X}$ \\
\hline Euxylophora paraensis & 0,1 & 0,2 & 1,82 & 0,12 & 0,03 & 0,17 & 0,36 & 0,49 & A & $\mathrm{X}$ & & $\mathrm{X}$ \\
\hline Parkia nítida & 0,08 & 0,16 & 3,27 & 0,22 & 0,02 & 0,1 & 0,26 & 0,48 & NA & & & $\mathrm{X}$ \\
\hline Micropholis sp. & 0,08 & 0,16 & 2,91 & 0,2 & 0,02 & 0,12 & 0,28 & 0,48 & $\mathrm{~T} \mathrm{~A}$ & $\mathrm{X}$ & & $\mathrm{X}$ \\
\hline Humiria balsamifera & 0,11 & 0,22 & 1,09 & 0,07 & 0,03 & 0,19 & 0,4 & 0,48 & A & $\mathrm{X}$ & & $\mathrm{X}$ \\
\hline Rheedia benthamiana & 0,06 & 0,11 & 2,18 & 0,15 & 0,02 & 0,13 & 0,24 & 0,39 & $\mathrm{NA}$ & & & $\mathrm{X}$ \\
\hline$N I-10$ & 0,06 & 0,13 & 2,18 & 0,15 & 0,02 & 0,11 & 0,24 & 0,39 & $\mathrm{TA}$ & $\mathrm{X}$ & & \\
\hline Inga paraensis & 0,07 & 0,14 & 2,55 & 0,17 & 0,01 & 0,07 & 0,21 & 0,38 & $\mathrm{~T}$ A & $\mathrm{X}$ & & $\mathrm{X}$ \\
\hline Laetia procera & 0,06 & 0,11 & 2,18 & 0,15 & 0,02 & 0,12 & 0,22 & 0,37 & $\mathrm{NA}$ & & $\mathrm{X}$ & $\mathrm{X}$ \\
\hline $\begin{array}{l}\text { Dimorphandra } \\
\text { multiflora }\end{array}$ & 0,05 & 0,09 & 1,82 & 0,12 & 0,03 & 0,16 & 0,25 & 0,37 & $\mathrm{NA}$ & & $\mathrm{X}$ & $\mathrm{X}$ \\
\hline
\end{tabular}

Valores apresentados em ordem decrescente de valor de importância (VI). DA = densidade absoluta (ind. ha $\left.{ }^{-1}\right) ; \mathrm{DR}=$ densidade relativa $(\%)$; FA= frequência absoluta; $\mathrm{FR}=$ frequência relativa; $\mathrm{DoA}=$ dominância absoluta $\left(\mathrm{m}^{2} \mathrm{ha}^{-1}\right) ; \mathrm{DoR}=$ dominância relativa; $\mathrm{VC}=$ valor de cobertura; $\mathrm{VI}=$ valor de importância e $\mathrm{Pi}$ = índice de Payandeh $(\mathrm{A}=$ agrupamento; $\mathrm{T} \mathrm{A}=$ tendência agrupamento; $\mathrm{N} \mathrm{A}=$ não agrupada $), \mathrm{NI}=$ não identificada. $\mathrm{TCF}=$ transição Cerrado Floresta, FTFDSm = Floresta de Terra Firme Densa Submontana e FTFDBP = Floresta de Terra Firme Densa de Baixo Platô. 
Tabela 1. Continuação.

\begin{tabular}{|c|c|c|c|c|c|c|c|c|c|c|c|c|}
\hline Espécie & DA & DR & FA & FR & DoA & DoR & $\mathrm{VC}$ & VI & $\mathbf{P i}$ & TCF & FTFDSm & FTFDBP \\
\hline Pouteria engleri & 0,06 & 0,13 & 1,82 & 0,12 & 0,02 & 0,1 & 0,23 & 0,35 & A & & & $\mathrm{X}$ \\
\hline Ocotea $\mathrm{sp} .3$ & 0,06 & 0,13 & 2,18 & 0,15 & 0,01 & 0,07 & 0,2 & 0,34 & T A & & $\mathrm{X}$ & $\mathrm{X}$ \\
\hline Chimarris turbinata & 0,06 & 0,13 & 1,45 & 0,1 & 0,02 & 0,09 & 0,22 & 0,32 & A & & & $\mathrm{X}$ \\
\hline Bertholletia excelsa & 0,04 & 0,07 & 1,45 & 0,1 & 0,03 & 0,14 & 0,22 & 0,31 & N A & & $\mathrm{X}$ & \\
\hline Guarea carinata & 0,06 & 0,11 & 2,18 & 0,15 & 0,01 & 0,05 & 0,16 & 0,3 & NA & & & $\mathrm{X}$ \\
\hline Pradosia praealta & 0,04 & 0,07 & 1,45 & 0,1 & 0,02 & 0,13 & 0,21 & 0,3 & NA & $\mathrm{X}$ & & $\mathrm{X}$ \\
\hline Ceiba pentandra & 0,05 & 0,09 & 1,82 & 0,12 & 0,01 & 0,07 & 0,16 & 0,28 & N A & & & $\mathrm{X}$ \\
\hline Caraipa grandiflora & 0,05 & 0,09 & 1,82 & 0,12 & 0,01 & 0,06 & 0,15 & 0,27 & NA & $\mathrm{X}$ & & $\mathrm{X}$ \\
\hline Mezilaurus itauba & 0,05 & 0,09 & 1,82 & 0,12 & 0,01 & 0,06 & 0,15 & 0,27 & N A & & & $\mathrm{X}$ \\
\hline Diplotropis sp. & 0,05 & 0,09 & 1,82 & 0,12 & 0,01 & 0,06 & 0,15 & 0,27 & N A & $\mathrm{X}$ & & $\mathrm{X}$ \\
\hline Brosimum lactescens & 0,05 & 0,09 & 1,82 & 0,12 & 0,01 & 0,05 & 0,14 & 0,27 & NA & $\mathrm{X}$ & & $\mathrm{X}$ \\
\hline Tapirira guianensis & 0,05 & 0,09 & 1,82 & 0,12 & 0,01 & 0,05 & 0,14 & 0,26 & N A & & & $\mathrm{X}$ \\
\hline $\begin{array}{l}\text { Trymatococcus } \\
\text { amazonicus }\end{array}$ & 0,04 & 0,07 & 1,45 & 0,1 & 0,02 & 0,09 & 0,16 & 0,26 & N A & & $\mathrm{X}$ & $\mathrm{X}$ \\
\hline Mouriri sagotiana & 0,04 & 0,07 & 1,45 & 0,1 & 0,01 & 0,06 & 0,14 & 0,23 & N A & $\mathrm{X}$ & & $\mathrm{X}$ \\
\hline Elizabetha bicolor & 0,05 & 0,09 & 1,45 & 0,1 & 0,01 & 0,04 & 0,13 & 0,23 & $\mathrm{~T} \mathrm{~A}$ & & $\mathrm{X}$ & $\mathrm{X}$ \\
\hline Schefflera morototoni & 0,04 & 0,07 & 1,45 & 0,1 & 0,01 & 0,06 & 0,13 & 0,23 & N A & $\mathrm{X}$ & & $\mathrm{X}$ \\
\hline Endopleura uchi & 0,04 & 0,07 & 1,45 & 0,1 & 0,01 & 0,05 & 0,13 & 0,22 & NA & & $\mathrm{X}$ & \\
\hline Swartzia racemosa & 0,04 & 0,07 & 1,45 & 0,1 & 0,01 & 0,05 & 0,13 & 0,22 & N A & $\mathrm{X}$ & & $\mathrm{X}$ \\
\hline Trichilia septentrionalis & 0,04 & 0,07 & 1,45 & 0,1 & 0,01 & 0,05 & 0,12 & 0,22 & NA & & & $\mathrm{X}$ \\
\hline Diplotropis purpurea & 0,04 & 0,07 & 1,45 & 0,1 & 0,01 & 0,05 & 0,12 & 0,22 & N A & $X$ & & $X$ \\
\hline Aspidosperma exalatum & 0,04 & 0,07 & 1,09 & 0,07 & 0,01 & 0,08 & 0,15 & 0,22 & $\mathrm{~T} \mathrm{~A}$ & & $\mathrm{X}$ & \\
\hline Guarea macrophylla & 0,03 & 0,05 & 1,09 & 0,07 & 0,01 & 0,08 & 0,13 & 0,21 & N A & & & $\mathrm{X}$ \\
\hline Swartzia panococo & 0,03 & 0,05 & 1,09 & 0,07 & 0,01 & 0,08 & 0,13 & 0,21 & N A & $\mathrm{X}$ & & $\mathrm{X}$ \\
\hline Pourouma minor & 0,04 & 0,07 & 1,45 & 0,1 & 0,01 & 0,03 & 0,1 & 0,2 & N A & & & $\mathrm{X}$ \\
\hline Xylopia benthamii & 0,03 & 0,05 & 1,09 & 0,07 & 0,01 & 0,08 & 0,13 & 0,2 & N A & & $\mathrm{X}$ & $X$ \\
\hline Cariniana micrantha & 0,03 & 0,05 & 1,09 & 0,07 & 0,01 & 0,06 & 0,11 & 0,19 & N A & & & $\mathrm{X}$ \\
\hline Anacardium spruceanum & 0,03 & 0,05 & 1,09 & 0,07 & 0,01 & 0,05 & 0,11 & 0,18 & N A & & $\mathrm{X}$ & $X$ \\
\hline Licania unguiculata & 0,03 & 0,05 & 1,09 & 0,07 & 0,01 & 0,05 & 0,1 & 0,18 & N A & $\mathrm{X}$ & & $\mathrm{X}$ \\
\hline Swartzia sp.1 & 0,03 & 0,05 & 1,09 & 0,07 & 0,01 & 0,05 & 0,1 & 0,17 & NA & $\mathrm{X}$ & & $\mathrm{X}$ \\
\hline Swartzia sp.3 & 0,03 & 0,05 & 1,09 & 0,07 & 0,01 & 0,04 & 0,1 & 0,17 & N A & $\mathrm{X}$ & & $\mathrm{X}$ \\
\hline Couratari sp. & 0,03 & 0,05 & 1,09 & 0,07 & 0,01 & 0,04 & 0,1 & 0,17 & N A & & & $\mathrm{X}$ \\
\hline Iryanthera juruensis & 0,03 & 0,05 & 1,09 & 0,07 & 0,01 & 0,04 & 0,09 & 0,17 & N A & & & $\mathrm{X}$ \\
\hline Zygia ramiflora & 0,01 & 0,02 & 0,36 & 0,02 & 0,02 & 0,12 & 0,14 & 0,17 & N A & & & $\mathrm{X}$ \\
\hline Macrolobium sp. & 0,03 & 0,05 & 1,09 & 0,07 & 0,01 & 0,04 & 0,09 & 0,16 & NA & & & $\mathrm{X}$ \\
\hline Bowdichia virgilioides & 0,03 & 0,05 & 1,09 & 0,07 & 0,01 & 0,04 & 0,09 & 0,16 & A & $\mathrm{X}$ & & $\mathrm{X}$ \\
\hline Pouteria sp.5 & 0,03 & 0,05 & 0,73 & 0,05 & 0,01 & 0,06 & 0,11 & 0,16 & N A & & & $\mathrm{X}$ \\
\hline
\end{tabular}

Valores apresentados em ordem decrescente de valor de importância (VI). DA = densidade absoluta (ind. ha ${ }^{-1}$ ); DR $=$ densidade relativa (\%); FA= frequência absoluta; $\mathrm{FR}=$ frequência relativa; $\mathrm{DoA}=$ dominância absoluta $\left(\mathrm{m}^{2} \mathrm{ha}^{-1}\right) ; \mathrm{DoR}=$ dominância relativa; $\mathrm{VC}=$ valor de cobertura; $\mathrm{VI}=$ valor de importância e $\mathrm{Pi}$ = índice de Payandeh ( $\mathrm{A}$ = agrupamento; $\mathrm{T} \mathrm{A}=$ tendência agrupamento; $\mathrm{N} \mathrm{A}=$ não agrupada $), \mathrm{NI}=$ não identificada. TCF = transição Cerrado Floresta, FTFDSm = Floresta de Terra Firme Densa Submontana e FTFDBP = Floresta de Terra Firme Densa de Baixo Platô. 
Tabela 1. Continuação.

\begin{tabular}{|c|c|c|c|c|c|c|c|c|c|c|c|c|}
\hline Espécie & DA & DR & FA & $\mathbf{F R}$ & DoA & DoR & $\mathrm{VC}$ & VI & $\mathbf{P i}$ & TCF & FTFDSm & FTFDBP \\
\hline Geissospermum vellosii & 0,03 & 0,05 & 1,09 & 0,07 & 0,01 & 0,03 & 0,08 & 0,16 & NA & & & $\mathrm{X}$ \\
\hline Ambelania acida & 0,03 & 0,05 & 1,09 & 0,07 & 0,01 & 0,03 & 0,08 & 0,16 & $\mathrm{NA}$ & & & $\mathrm{X}$ \\
\hline Planchonella sp. & 0,02 & 0,04 & 0,73 & 0,05 & 0,01 & 0,06 & 0,1 & 0,14 & NA & & & $\mathrm{X}$ \\
\hline Protium apiculatum & 0,03 & 0,05 & 0,73 & 0,05 & 0,01 & 0,04 & 0,09 & 0,14 & A & $\mathrm{X}$ & & $\mathrm{X}$ \\
\hline Abarema auriculata & 0,03 & 0,05 & 0,73 & 0,05 & 0,01 & 0,03 & 0,09 & 0,14 & A & $\mathrm{X}$ & $\mathrm{X}$ & $\mathrm{X}$ \\
\hline Licania heteromorpha & 0,04 & 0,07 & 0,36 & 0,02 & 0,01 & 0,04 & 0,11 & 0,13 & A & & & $\mathrm{X}$ \\
\hline Mezilaurus duckei & 0,02 & 0,04 & 0,73 & 0,05 & 0,01 & 0,05 & 0,08 & 0,13 & $\mathrm{NA}$ & & $\mathrm{X}$ & $\mathrm{X}$ \\
\hline Tapura guianensis & 0,02 & 0,04 & 0,73 & 0,05 & 0,01 & 0,05 & 0,08 & 0,13 & N A & $\mathrm{X}$ & & $\mathrm{X}$ \\
\hline Siparuna sp. & 0,02 & 0,04 & 0,73 & 0,05 & 0,01 & 0,03 & 0,06 & 0,11 & N A & & & $\mathrm{X}$ \\
\hline Protium sagotianum & 0,02 & 0,04 & 0,73 & 0,05 & 0,01 & 0,03 & 0,06 & 0,11 & N A & & & $\mathrm{X}$ \\
\hline $\begin{array}{l}\text { Aspidosperma } \\
\text { carapanauba }\end{array}$ & 0,02 & 0,04 & 0,73 & 0,05 & 0 & 0,03 & 0,06 & 0,11 & N A & $\mathrm{X}$ & & \\
\hline Candolleodendron sp. & 0,02 & 0,04 & 0,73 & 0,05 & 0 & 0,03 & 0,06 & 0,11 & N A & & & $X$ \\
\hline Diospyros praetermissa & 0,02 & 0,04 & 0,73 & 0,05 & 0 & 0,02 & 0,06 & 0,11 & NA & & & $\mathrm{X}$ \\
\hline Tabernaemontana sp. & 0,02 & 0,04 & 0,73 & 0,05 & 0 & 0,02 & 0,06 & 0,11 & NA & & & $X$ \\
\hline Diospyros sp.2 & 0,02 & 0,04 & 0,73 & 0,05 & 0 & 0,02 & 0,06 & 0,1 & $\mathrm{NA}$ & & & $\mathrm{X}$ \\
\hline Pourouma villosa & 0,02 & 0,04 & 0,73 & 0,05 & 0 & 0,02 & 0,05 & 0,1 & N A & & & $\mathrm{X}$ \\
\hline Ptychopetalum olacoides & 0,02 & 0,04 & 0,73 & 0,05 & 0 & 0,02 & 0,05 & 0,1 & NA & & $\mathrm{X}$ & $\mathrm{X}$ \\
\hline Croton sp. & 0,02 & 0,04 & 0,73 & 0,05 & 0 & 0,02 & 0,05 & 0,1 & N A & & & $\mathrm{X}$ \\
\hline Tovomita sp. & 0,02 & 0,04 & 0,73 & 0,05 & 0 & 0,02 & 0,05 & 0,1 & N A & & & $\mathrm{X}$ \\
\hline Pouteria sp.3 & 0,01 & 0,02 & 0,36 & 0,02 & 0,01 & 0,03 & 0,05 & 0,08 & NA & $\mathrm{X}$ & & \\
\hline $\begin{array}{l}\text { Eriotheca } \\
\text { longipedicellata }\end{array}$ & 0,01 & 0,02 & 0,36 & 0,02 & 0 & 0,02 & 0,04 & 0,07 & N A & & & $\mathrm{X}$ \\
\hline Eperua sp. & 0,01 & 0,02 & 0,36 & 0,02 & 0 & 0,02 & 0,04 & 0,06 & N A & & & $\mathrm{X}$ \\
\hline Oxandra acuminata & 0,01 & 0,02 & 0,36 & 0,02 & 0 & 0,02 & 0,04 & 0,06 & N A & & & $X$ \\
\hline Protium pilosum & 0,01 & 0,02 & 0,36 & 0,02 & 0 & 0,02 & 0,04 & 0,06 & NA & & & $\mathrm{X}$ \\
\hline Guarea kunthiana & 0,01 & 0,02 & 0,36 & 0,02 & 0 & 0,02 & 0,04 & 0,06 & NA & & & $X$ \\
\hline Sloanea sp. & 0,01 & 0,02 & 0,36 & 0,02 & 0 & 0,02 & 0,04 & 0,06 & $\mathrm{NA}$ & & & $\mathrm{X}$ \\
\hline Mouriri apiranga & 0,01 & 0,02 & 0,36 & 0,02 & 0 & 0,02 & 0,04 & 0,06 & N A & $\mathrm{X}$ & & $\mathrm{X}$ \\
\hline Trichilia sp. & 0,01 & 0,02 & 0,36 & 0,02 & 0 & 0,02 & 0,04 & 0,06 & NA & $\mathrm{X}$ & & \\
\hline Theobroma speciosum & 0,01 & 0,02 & 0,36 & 0,02 & 0 & 0,02 & 0,03 & 0,06 & N A & $\mathrm{X}$ & & \\
\hline Hortia excelsa & 0,01 & 0,02 & 0,36 & 0,02 & 0 & 0,01 & 0,03 & 0,06 & N A & & & $\mathrm{X}$ \\
\hline Quararibea sp. & 0,01 & 0,02 & 0,36 & 0,02 & 0 & 0,02 & 0,03 & 0,06 & N A & $\mathrm{X}$ & & \\
\hline Cordia exaltata & 0,01 & 0,02 & 0,36 & 0,02 & 0 & 0,01 & 0,03 & 0,05 & N A & $\mathrm{X}$ & & \\
\hline Bellucia grossularioides & 0,01 & 0,02 & 0,36 & 0,02 & 0 & 0,01 & 0,03 & 0,05 & N A & & & $\mathrm{X}$ \\
\hline Henriettea spruceana & 0,01 & 0,02 & 0,36 & 0,02 & 0 & 0,01 & 0,03 & 0,05 & N A & $\mathrm{X}$ & & \\
\hline Pouteria opposita & 0,01 & 0,02 & 0,36 & 0,02 & 0 & 0,01 & 0,03 & 0,05 & N A & & & $\mathrm{X}$ \\
\hline Pouteria sp.1 & 0,01 & 0,02 & 0,36 & 0,02 & 0 & 0,01 & 0,03 & 0,05 & NA & & & $\mathrm{X}$ \\
\hline
\end{tabular}


Tabela 1. Continuação.

\begin{tabular}{|c|c|c|c|c|c|c|c|c|c|c|c|c|}
\hline Espécie & DA & DR & FA & FR & DoA & DoR & VC & VI & $\mathbf{P i}$ & TCF & FTFDSm & FTFDBP \\
\hline Lacistema grandifolium & 0,01 & 0,02 & 0,36 & 0,02 & 0 & 0,01 & 0,03 & 0,05 & NA & & & $\mathrm{X}$ \\
\hline Pouteria macrophylla & 0,01 & 0,02 & 0,36 & 0,02 & 0 & 0,01 & 0,03 & 0,05 & NA & & $\mathrm{X}$ & \\
\hline Sapium populifolium & 0,01 & 0,02 & 0,36 & 0,02 & 0 & 0,01 & 0,03 & 0,05 & $\mathrm{NA}$ & & & $\mathrm{X}$ \\
\hline Miconia pubipetala & 0,01 & 0,02 & 0,36 & 0,02 & 0 & 0,01 & 0,03 & 0,05 & NA & & & $\mathrm{X}$ \\
\hline NI-4 & 0,01 & 0,02 & 0,36 & 0,02 & 0 & 0,01 & 0,03 & 0,05 & NA & $\mathrm{X}$ & $\mathrm{X}$ & \\
\hline Xylopia nitida & 0,01 & 0,02 & 0,36 & 0,02 & 0 & 0,01 & 0,03 & 0,05 & $\mathrm{NA}$ & & & $\mathrm{X}$ \\
\hline Ocotea petalanthera & 0,01 & 0,02 & 0,36 & 0,02 & 0 & 0,01 & 0,03 & 0,05 & $\mathrm{NA}$ & & & X \\
\hline Total & 50,7 & 100 & 1486 & 100 & 17 & 100 & 200 & 300 & & & & \\
\hline
\end{tabular}

Valores apresentados em ordem decrescente de valor de importância $(\mathrm{VI}) . \mathrm{DA}=$ densidade absoluta (ind. ha $\left.{ }^{-1}\right) ; \mathrm{DR}=$ densidade relativa $(\%)$; $\mathrm{FA}=$ frequência absoluta; $\mathrm{FR}=$ frequência relativa; $\mathrm{DoA}=$ dominância absoluta $\left(\mathrm{m}^{2} \mathrm{ha}^{-1}\right) ; \mathrm{DoR}=$ dominância relativa; $\mathrm{VC}=$ valor de cobertura; $\mathrm{VI}=$ valor de importância e $\mathrm{Pi}$ = índice de Payandeh $(\mathrm{A}=$ agrupamento; $\mathrm{T} \mathrm{A}=$ tendência agrupamento; $\mathrm{N} \mathrm{A}=$ não agrupada $), \mathrm{NI}=$ não identificada. TCF $=$ transição Cerrado Floresta, FTFDSm = Floresta de Terra Firme Densa Submontana e FTFDBP = Floresta de Terra Firme Densa de Baixo Platô.

Essas espécies com maiores densidades podem estar relacionadas com as características favoráveis do ambiente (Holanda et al., 2010) ou até mesmo o estágio de sucessão da área. Assim, o monitoramento da dinâmica florestal pode revelar o comportamento das espécies ao longo do tempo e identificar possíveis fatores que favorecem o ingresso e manutenção destas espécies.

Valencia et al. (1994) em estudo realizado por 1 ha de floresta de terra firme, na Amazônia Equatoriana, em que foram computadas 307 espécies com pelo menos $10 \mathrm{~cm}$ de DAP, também relataram a ocorrência da Eschweilera aff. coriacea (Lecythidaceae) como uma das espécies com maior abundância, sendo as famílias Leguminosae, Lauraceae, Sapotaceae, Moraceae e Burseraceae as mais diversas.

As espécies que apresentaram maiores frequências relativas, em ordem decrescente, foram: Goupia glabra, Carapa guianensis, Eschweilera sp., Eschweilera coriacea e Vochysia sp.1, sendo espécies amostradas em toda área. Neste sentido, se faz necessário realizar estudos de monitoramentos para obter taxas de recrutamento e mortalidade e dispersão, para inferir sobre à sua distribuição. Espécies com maiores distribuições (amplitude de nicho), apresentam a capacidade de explorar diferentes recursos, além de uma maior tolerância às alterações em seu ambiente (Gaston \& Fuller, 2007), podem ter maiores chances de se adaptarem a mudanças naturais ou antrópicas ocorridas no ambiente (Laurance, 2004), sofrendo menor risco de extinção local.

Goupia glabra apresentou valores mais altos de valor de cobertura (VC) e de valor de importância (VI), sendo que as demais espécies trocaram algumas posições quando considerado o VC ou o VI, porém, não foram consideradas grandes alterações.

Segundo Oliveira \& Amaral (2004), o valor de importância (VI) é uma boa ferramenta para gestão da floresta, pois funciona como um indicador da importância ecológica. Neste sentido, tal importância reflete a influência das espécies mais frequentes e dominantes nos processos de equilíbrio da flora e manutenção da fauna, pois podem fornecer abrigo e alimentação para o conjunto de seres vivos presentes no habitat.

O alto VI de Goupia glabra (cupiúba) pode ser explicado pelos seus valores de diâmetro, que são representados na dominância relativa e seu valor de número de indivíduos (densidade relativa). Matos \& Amaral (1999), em uma Floresta de Terra Firme no Amazonas, também encontraram Goupia glabra como a espécie mais importante, principalmente pelos valores de dominância relativa. No estudo desses autores a espécie foi classificada como de interesse econômico. Essa espécie se enquadra no grupo de espécies de reconhecido valor madeireiro e características tecnológicas para indústria madeireira (Pinheiro et al., 2007; Instituto Natureza Amazônica, 2010). Segundo Lorenzi (2002) a madeira de Goupia glabra apresenta alta densidade $\left(0,87 \mathrm{~g} \mathrm{~cm}^{-3}\right)$, é dura e bastante resistente ao ataque de organismos xilófagos, podendo ser indicada para construção naval e civil.

Outra espécie com maior valor de importância foi Carapa guianensis (andiroba) que apresentou valores relativamente uniformes de $\mathrm{DR}=2,73 ; \mathrm{FR}=2,55$; $\mathrm{DoR}=2,17$, sendo possível inferir que esta espécie está 
menos susceptível ao esgotamento genético nesse estágio da sucessão, devido aos valores uniformes (Matos \& Amaral, 1999; Oliveira et al., 2008), porém, estudos de dinâmica florestal podem auxiliar para a confirmação dessas hipóteses. Segundo Ferraz et al. (2002) Carapa guianensis é uma espécie de uso múltiplo, sendo que a madeira e o óleo extraído das sementes são os produtos mais importantes.

A composição florística e estrutura horizontal revelou que a área é rica em número de famílias, gêneros e espécies. Resultados semelhantes foram encontrados em outros trabalhos desenvolvidos na Amazônia (Oliveira \& Amaral, 2004; Francez et al., 2007; Alves \& Miranda, 2008; Oliveira et al., 2008, Pereira et al., 2011). Portanto, considerando a importância de muitas dessas espécies tanto do ponto de vista ecológico quanto econômico para a região Amazônica, estudos mais específicos sobre essas populações são necessários.

Em conformidade com o padrão de distribuição espacial calculado pelo índice de Payandeh (Pi) as espécies na Floresta de Terra Firme no estado do Amapá apresentaram a classificação: não agrupamento ou aleatória (47,26\%), tendência ao agrupamento (33,33\%) e $19,40 \%$ indicam agrupamento (Figura 2).

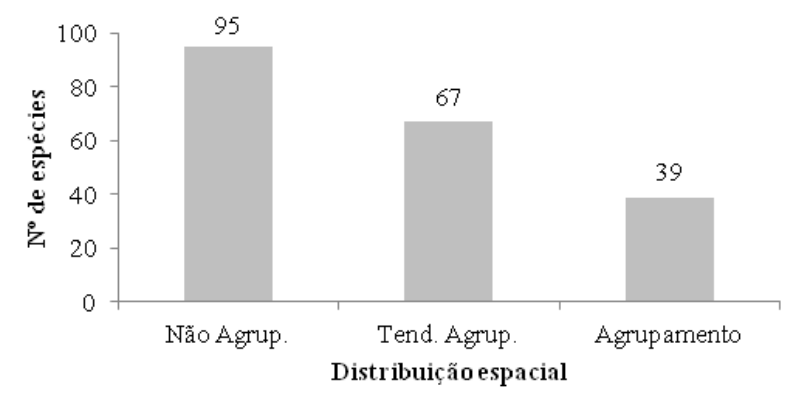

Figura 2. Distribuição espacial das espécies para a área de estudo no estado do Amapá. Distribuição não agrupada, tendência ao agrupamento e distribuição em agrupamento.

Na tipologia FTFDBP, 44 espécies $(23,4 \%)$ foram classificadas com distribuição espacial agrupada, 96 $(51,06 \%)$ com distribuição não agrupada e 48 (25,53\%) com tendência ao agrupamento; na tipologia FTFDSm, 48 espécies $(37,5 \%)$ mostraram-se agrupadas, 64 (50\%) não agrupadas e $16(12,5 \%)$ com tendência ao agrupamento e na tipologia TCF 20 espécies (19,2\%) mostraram-se agrupadas, 69 (66,3\%) não agrupadas e $15(14,4 \%)$ com tendência ao agrupamento.

Entre a principal causa que provavelmente favorece a distribuição aleatória, cita-se: diferentes processos dispersivos sem um padrão definido, desta forma, as espécies ocorrem mais espalhadas na vegetação, com menores valores das médias no número de indivíduos e baixos valores de Pi (geralmente menores que um) e podem, em determinados casos, apresentarem problemas de regeneração natural (Nascimento et al., 2001). A distribuição aleatória também pode estar relacionada com baixa densidade de algumas espécies e o nível de inclusão utilizado neste estudo (Bruzinga et al., 2013), visto que nesse trabalho foi verificado que $12,43 \%$ das espécies encontradas na amostra apresentaram apenas um indivíduo.

Entre as dez espécies de maior VI, sete apresentaram a classificação de Pi em agrupamento e três espécies com tendência ao agrupamento. De acordo com Scolforo (1998) a maioria das espécies que compõem uma floresta nativa tem o padrão de distribuição agregado. Para Leite (2001) fatores abióticos como o tipo de solo, estresse hídrico, altitude, intensidade luminosa e fatores bióticos como polinizadores, dispersores e espécies competidoras são algumas das variáveis encontradas capazes de afetar o padrão de distribuição espacial de uma espécie. Begon et al. (2007) relata que a explicação evolutiva mais simples para a distribuição agregada é que os organismos se agregam, quando e onde encontram condições favoráveis à reprodução e à sua sobrevivência. Segundo Cox \& Moore (2011), mesmo dentro de um habitat, as espécies podem ocupar micro-habitats diferentes, por estarem cercadas com diferentes condições físicas, ou com falta de recursos que não permitem sua sobrevivência.

Em ambientes heterogêneos o desenvolvimento dos indivíduos arbóreos ocorre com preferência por sítio, luminosidade e fertilidade (Bleher \& BöhningGaese, 2001; Nascimento et al., 2001; Perry \& Dixon, 2002; Brown \& Lomolino, 2006) ou pode estar ligada ao processo de dispersão, em que a regeneração ocorre próxima à planta mãe (Leite, 2001) e assim, as populações tendem a ser agrupadas.

\section{Conclusões}

A área florestal analisada apresenta características de floresta preservada. A riqueza de espécies entre as tipologias florestais foi separada em dois grupos. A tipologia Floresta de Terra Firme Densa de Baixo Platô contribui com maior número de espécies. As espécies de maior valor de importância na área estudada foram 
Goupia glabra e Carapa guianensis. A maioria das espécies de maior VI apresentam distribuição espacial na forma de manchas (agrupamento).

\section{Rereferências}

ALVES, J. C. Z. O; MIRANDA, I. S. Análise da estrutura de comunidades arbóreas de uma floresta amazônica de Terra Firme aplicada ao manejo florestal. Acta Amazônica, Manaus, v. 38, n. 4, p. 657-666, 2008.

AMAPÁ. Instituto Estadual de Florestas do Amapá. Plano anual de outorga florestal 2010. Macapá: 2009. 37 p.

AMAPÁ. Instituto Estadual de Florestas do Amapá. Plano anual de outorga florestal 2014. Macapá, 2013. 46 p.

ANGIOSPERM PHYLOGENY GROUP. An update of the angiosperm phylogeny group classification for orders and families of flowering plants:APG II. Botanical Journal of the Linnean Society, London, v. 141, p. 399-436, 2003.

BATISTA, A. P. B.; SANTOS, V. S.; APARÍCIO, W. C. S.; APARÍCIO, P. S.; SILVA, D. A. S. Similaridade e gradientes de riqueza florística em uma floresta de várzea na cidade de Macapá. Revista Verde, Mossoró, v. 8, n. 4, p. 152-158, 2013.

BEGON, M.; HARPER, J. L.; TOWHSEND, C. R. Fundamentos em ecologia. Porto Alegre: Artmed, 2007. 592 p.

BROWN, J. H.; LOMOLINO, M. V. Biogeografia. 2. ed. Ribeirão Preto: FUNPEC, 2006.

BLEHER, B.; BÖHNING-GAESE, K. Consequences of frugivore diversity for seed dispersal, seedling establishment and the spatial pattern of seedlings and trees. Oecologia, Berlin, v. 129, p. 385394, 2001.

BRUZINGA, J. S.; OLIVEIRA, M. L. R.; MACHADO, E. L. M.; LEITE, H. G.; PEREIRA, I. M.; NOGUEIRA, G. S. Distribuição espacial de indivíduos adultos de Pequi. Scientia Forestalis, Piracicaba, v. 42, n. 98, p. 249-256, 2013.

CIENTEC. Mata nativa 2: manual do usuário. Viçosa, MG, 2006. $126 \mathrm{p}$.

COSTA JUNIOR, R. F; FERREIRA, R. L. C; RODAL, M. J. N.; FELICIANO, A. L. P.; MARAGON, L. C.; SILVA, W. C. Estrutura fitossociológica do componente arbóreo de um fragmento de floresta ombrófila densa na mata sul de Pernambuco, nordeste do Brasil. Ciência Florestal, Santa Maria, RS, v.18, n. 2, p. 173-183, 2008.

COX, C. B.; MOORE, P. D. Biogeografia: uma abordagem ecológica e evolucionária. 7. ed. Rio de Janeiro: LTC, 2011.

DRUMMOnD, J. A.; DIAS, T. C. A. C.; BRITO, D. M. C. Atlas das Unidades de Conservação do Estado do Amapá. Macapá, AP: MMA/IBAMA; SEMA/IEF/IPCT, 2008. 128 p.

FELFILI, J. M.; REZENDE, R. P. Conceitos e métodos em fitossociologia. Brasília, DF: Ed da UNB, 2003. v. 5. 68 p.

FERRAZ, I. D. K.; CAMARGO, J. L. C.; SAMPAIO, P. T. B. Sementes e plântulas de andiroba (Carapa guianensis Aubl. e Carapa procera, D.C): aspectos botânicos, ecológicos e tecnológicos. Acta Amazônica, Manaus, v. 32, n. 4, p. 647-660, 2002.
FRANCEZ, L. M. B.; CARVALHO, J. O. P.; JARDIM, F. C. S. Mudanças ocorridas na composição florística em decorrência da exploração florestal em uma área de floresta de Terra Firme na região de Paragominas, PA. Acta Amazônica, Manaus, v. 37, n. 2 , p. 219-228. 2007.

GASTON, K. J.; FULLER, R. A. Biodiversity and extinction: losing the common and the widespread. Progress in Physical Geography, v. 31, p. 213-225, 2007.

HOLANDA, A. C.; FELICIANO, A. L. P.; MARANGON, L. C.; SANTOS, M. S.; MELO, C. L. S. M. S.; PESSOA, M. M. L. Estrutura de espécies arbóreas sob efeito de borda em um fragmento de floresta estacional semidecidual em Pernambuco. Revista Árvore, Viçosa, MG, v. 34, n. 1, p. 103-114, 2010.

IBGE. Manual técnico da vegetação brasileira: sistema fitogeográfico, inventário das formações florestais e campestres, técnicas e manejo de coleções botânicas, procedimentos para mapeamentos. 2. ed. rev. e amp. Rio de Janeiro, 2012. 275 p.

INSTITUTO NATUREZAAMAZÔNICA. Relatório do inventário florestal amostral da Floresta Estadual do Amapá. 2010. Não publicado.

KOTTEK, M.; GRIESER,J.; BECK, C.; RUDOLF, B.; RUBEL, F. World Map of the Köppen-Geiger climate classification updated. Meteorologishe Zeitschrift, v. 15, p. 259-263, 2006.

LAURANCE, W. F. Forest-climate interactions in fragmented tropical landscapes. Philosophical Transactions of the Royal Society of London, Series B, v. 359, p. 345-352, 2004.

LEITE, E. J. Spatial distribution patterns of riverine forest taxa in Brasília, Brazil. Forest Ecology and Management, Amsterdam, v. 140, p. 257-264, 2001.

LORENZI, H. Árvores brasileiras: manual de identificação e cultivo de plantas arbóreas nativas do Brasil. 2. ed. Nova Odessa: Instituto Plantarum, 2002.

MATOS, F. D.; AMARAL, I. L. Análise ecológica de um hectare em floresta ombrófila densa de terra firme, estrada de várzea, Amazonas Brasil. Acta Amazônica, Manaus, v. 29, n. 3, p. 365-379. 1999.

NASCIMENTO, A. R. T.; LONGHI, S. J.; BRENA, D. A. Estrutura e padrões de distribuição espacial de espécies arbóreas em uma amostra de floresta ombrófila mista em Nova Prata, RS, Ciência Florestal, Santa Maria, RS, v. 11, n. 1, p. 105-119, 2001.

OKSANEN, J.; BLANCHET, F. G.; KINDT, R.; LEGENDRE, P.; MINCHIN, P. R.; O'HARA, R. B.; SIMPSON, P. S.; STEVENS, M. H. H.; WAGNER, H. Vegan: Community Ecology Package: R package version 2.0-9. 2013.

OLIVEIRA, A. N.; AMARAL, I. L.; RAMOS, M. B. P.; NOBRE, A. D.; COUTO, L. B.; SAHDO, R. M. Composição e diversidade florístico-estrutural de um hectare de floresta densa de terra firme na Amazônia Central, Amazonas, Brasil. Acta Amazônica, Manaus, v. 38, n. 4, p. 627-642, 2008.

OLIVEIRA, A. N.; AMARAL, I. L; Florística e fitossociologia de uma floresta de vertente na Amazônia Central, Amazonas, Brasil. Acta Amazônica, Manaus, v. 34, n. 1, 2004.

PEREIRA, L. A.; SOBRINHO, F. A. P.; COSTA NETO, S. V. Florística e estrutura de uma mata de terra firme na Reserva de 
Desenvolvimento Sustentável Rio Iratapuru, Amapá, Amazônia Oriental, Brasil. Floresta, Curitiba, v. 41, n. 1, p. 113-122, 2011.

PEREIRA, L. A.; SENA, K. S.; SANTOS, M. R; COSTA NETO, S. V. Aspectos florísticos da Flona do Amapá e sua a importância na conservação da biodiversidade. Revista Brasileira de Biociências, Porto Alegre, v. 5, n. 2, p. 693-695, 2007.

PERRY, J. N.; DIXON, P. M. A new method to measure spatial association for ecological count data. Ecoscience, Sainte-Foy, v. 9, p. 133-141, 2002.

PINHEIRO, K. A. O.; CARVALHO, J. O. P. D.; QUANZ, B.; FRANCEZ, L. M. D. B.; SCHWATZ, G. Fitossociologia de uma área de preservação permanente no leste da Amazônia: indicação de espécies para recuperação de áreas alteradas. Floresta, Curitiba, v. 37, n. 2, p. 175-187, 2007.

RABELO, B. V. (Coord). Macrodiagnóstico do Estado do Amapá: primeira aproximação do ZEE. 3. ed. Macapá: Instituto de Pesquisas Científicas e Tecnológicas do Estado do Amapá, Equipe Técnica do Programa Zoneamento Ecológico Econômico do Estado do Amapá, 2008. 139 p.
R CORE TEAM. R: a language and environment for statistical computing. R Foundation for Statistical Computing. Vienna, 2013. Disponível em: <http://www.R-project.org/>. Acesso em: 15 maio 2014.

SCOLFORO, J. R. S. Manejo florestal. Lavras: UFLA/ FAEPE, 1998.

SOUZA, A. L.; FERREIRA, R. L. C.; XAVIER, A. Análise de agrupamento aplicada à área florestal. Viçosa, MG: SIF, 1997. $109 \mathrm{p}$.

STATSOFT. Statistica: Data Analysis Software System: version 8. 2012.

TROPICOS. Missouri Botanical Garden. Disponível em: <http:// www.tropicos.org/>. Acesso em: 15 maio 2014.

VAlENCIA, R.; BALSLEV, H.; PAZ Y MINO, G. C. High tree alpha-diversity in Amazonian Ecuador. Biodiversity and Conservation, New Zealand, v. 3, p. 21-28, 1994. 
